\title{
Framework to Support Mobility Context Awareness in Cellular Networks
}

\author{
Nandish P. Kuruvatti, Hans D. Schotten \\ Institute for Wireless Communications and Navigation \\ University of Kaiserslautern \\ Email: \{kuruvatti,schotten\}@eit.uni-kl.de
}

\begin{abstract}
Mobile communication is arguably the most ubiquitously used technology in contemporary world, evolving towards its fifth generation (5G). The key challenges being faced by present day mobile communication are growing number of mobile users and subsequent high traffic volume posed by them. Providing uniform service quality and best quality of experience (QoE) in such dense scenarios is a major motive of 5G. Context awareness is a concept of extracting information from the user and his environment, and utilizing it to optimize user performance. Context awareness is recognized as one of the key pillars in enabling uniform quality of experience for mobile users. For instance, predicting the next cell for user transition, predicting the crowd formation in a cell etc., will assist the basestation to reserve or manage resources and prepare the cell well in advance for a future event, targeting to provide uninterrupted and uniform QoE. This paper investigates context aware procedures with a focus on user mobility, finds commonalities among different procedures and proposes a general framework to support mobility context awareness. The new information and interfaces which are required from various entities (e.g., vehicular infrastructure) are discussed. Further, a context aware resource allocation algorithm is designed, exploiting new interfaces and information arising from vehicular infrastructure. Simulation results demonstrate improvements in user throughput, thus validating the requirement of new interfaces.
\end{abstract}

Index Terms- 5G, Context awareness, mobility, cell transition prediction, route prediction

\section{INTRODUCTION}

Mobile communication has become a widely used technology in day to day life, with expanding horizon of its applications ever since its inception. Mobile communication has undergone evolution through several generations of technology and is currently on its way towards fifth generation (5G). Over several years, there has been a dramatic increase in the number of mobile users across the globe [1]. It is reported that, by the year 2020 there will be more than 50 billion connected devices [1]. Further, mobile broadband services are growing in popularity along with supporting hand-held and personal devices [2]. These factors have led to a avalanche of traffic volume growth and it is anticipated that mobile traffic volume will be at least a 1000 times larger in year 2020 than the present [1][2]. Accommodating such large number of users and resulting traffic volume is a key challenge in 5G. Providing uninterrupted uniform service quality and best QoE irrespective of high mobility and high traffic volume are major concerns to be addressed in 5G [3].

Context information is the information that enables the perception of states and situations of network entities (e.g., network nodes, terminals, users, etc.) and their interactive relations [4]. The radio network becomes context aware when context information is utilized to assist and optimize the operation of the network. The usage of context information and building context awareness were first studied in the field of pervasive computing. Relevant information such as user identity, location etc., were collected from environment and fed to computing system. Based on these information, the system would adapt accordingly to optimize its performance. In recent times, mobile and wireless systems have started to exploit the concepts of context awareness, making the radio network able to adapt its configurations or actions to the changing application and physical environment. Context awareness in cellular networks is recognized as an important pillar in tackling key challenges in 5G mobile communication [4].

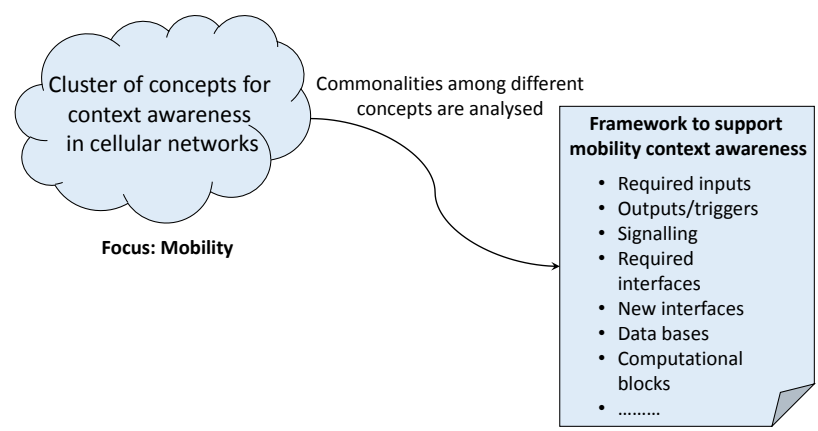

Fig. 1. Motivation

The work presented here deals specifically with context awareness in cellular networks, centered around mobility of users. Mobility prediction has been a key component in building context awareness. By anticipating/learning mobility behavior of the user, it is possible to design various context aware resource management schemes, handover procedures, cell activation/deactivation schemes etc. In literature, there are several related schemes that exploit user mobility behavior to build context awareness. This work proposes a framework to support mobility context awareness in cellular network by analyzing the commonalities among several context aware schemes. This paper also investigates on the set of required 
inputs, signaling and interfaces. Further, a context aware resource allocation scheme is designed that makes use of new interfaces and information arising from vehicular infrastructure. The simulation results show substantial improvements in throughput of the user, thereby supporting the need for new interfaces.

The remainder of this paper is organized as follows: Section II deals with related work, outlining context aware procedures focusing on mobility aspects. Section III discusses framework for enabling mobility context awareness, investigates required inputs, signaling and interfaces, presents supporting simulation results, and Section IV provides a conclusion and indicates future work.

\section{RELATED WORK}

This section outlines some of the major context aware schemes in the literature that are built using mobility aspects of user. Prediction of future user location (e.g., cells, routes etc) plays a key role in designing efficient context aware schemes. Various resource reservation/management schemes, handover optimization procedures are designed by utilizing the mobility information of the user. Mobility prediction can be briefly classified into two groups:

1) History based: The user's next cell or path is predicted based on the statistics of user's mobility. The mobility history of the user is recorded and probability of user transition into next cell is derived. The common methods of deriving probability of transition into next cell involve Markov chain model [5], hidden markov model [6], neural networks and machine learning [7], route clustering [8] etc.

2) Measurement based: These schemes do not rely on the user mobility history rather they derive probability of user transition to next cell based on real time measurements (e.g., RSSI, geometry, user angle, distance etc). [9], [10] make use of signal strength (RSSI, geometry) in $\mathrm{dB}$ to predict next cell, where as, [11] relies on user angle and distances to predict the next cell.

These mobility prediction schemes are then used to proactively reserve resources [5] [6], trigger load balancing (LB) [11] or activate/deactivate small cells [10]. Thus, mobility prediction can be seen as a driving force for context awareness in cellular networks.

\section{Mobility Context Awareness Framework}

Based on commonalities among various context aware schemes discussed in section II, a general framework to support mobility context awareness is proposed in this section. The set of required information, involved signaling and interfaces are outlined. Further, a context aware resource allocation scheme is presented that makes use of information arising from vehicular infrastructure. Simulation results are also discussed in this section.

\section{A. Required Information Set}

The common information set required as input by the mobility context aware schemes are listed in this section.

- User position: This information can be in the form of $(\mathrm{x}, \mathrm{y}, \mathrm{z})$ co-ordinates and could be obtained from global positioning system (GPS) or network assisted positioning.

- User velocity: This information can be obtained by doppler measurements or can be obtained from speedometer of vehicle by using proper interfacing from vehicular infrastructure to cellular network.

- User geometry $(\mathrm{dB})$ : Measured at the user terminal.

- Neighboring cells list (NCL): Maintained by operations support system (OSS) [13].

- Route maps: Similar to NCL but has information about roads, cross roads, possible coverage holes in them.

\section{B. Information from Vehicular Infrastructure}

In addition to the information set discussed in section III-A, we propose to extract and exploit the following information from vehicular infrastructure to enhance the mobility prediction and context awareness.

- Origin: The initial position (location) from where the user started his journey.

- Destination: The final position (location) to where the user intends to travel.

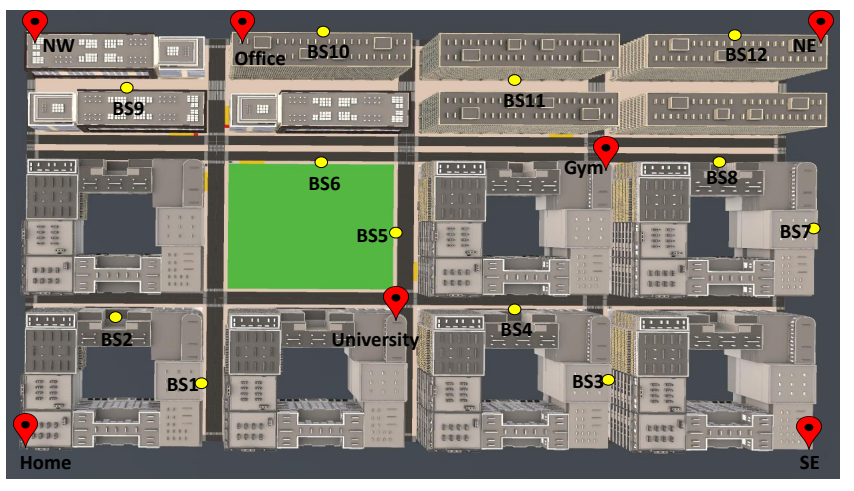

Fig. 2. Simulation Scenario

Consider the top view of 3D simulation scenario depicted in figure 2, referred to as Madrid grid [12]. There are 12 micro base stations in the scenario and it also includes parks, buildings, roads and crossroads. There are 7 landmarks that can act as origin and destination of the user (Home, university, office, gym, north west(NW), north east (NE) and south west(SW)). The probability of transition of a user from base station $B S_{n}$ to $B S_{n+1}$ based on markov based next cell prediction [5] can be obtained by,

$$
P\left(B S_{n} \rightarrow B S_{n+1}\right)=\frac{N\left(B S_{n} \rightarrow B S_{n+1}\right)}{N\left(B S_{n}\right)}
$$

Where, $B S_{n} \rightarrow \mathrm{BS}_{n+1}$ indicates transition from cell $l_{n}$ to cell $_{n+1}, N($.$) indicates the number of times. Thus, by using$ mobility statistics, probability of transition into a next cell can 
be obtained as the ratio between; number of times user in cell $_{n}$ transited to cell $_{n+1}$ and number of times user was found in $\mathrm{cell}_{n}$.

This model can be enhanced by using additional context information such as origin of the user $(\mathrm{O})$ and his destination (D). The resulting probability equations are

$$
\begin{gathered}
P\left(B S_{n} \rightarrow B S_{n+1} / O\right)=\frac{N\left(B S_{n} \rightarrow B S_{n+1} / O\right)}{N\left(B S_{n} / O\right)} \\
P\left(B S_{n} \rightarrow B S_{n+1} / O \& D\right)=\frac{N\left(B S_{n} \rightarrow B S_{n+1} / O \& D\right)}{N\left(B S_{n} / O \& D\right)}
\end{gathered}
$$

In eq.2, the statistics of the user only when he originated from a particular origin are considered, where as, in eq. 3 user statistics when originated from a specific origin and traveling to a specific destination are considered.

Similarly, the next route (road) of a user can be predicted before he reaches the cross road. This is also analogous to predicting the turn probability at each cross road. The probability of transition of a user from road $R_{n}$ to $R_{n+1}$ based on markov based route prediction [5] is given by,

$$
P\left(R_{n} \rightarrow R_{n+1}\right)=\frac{N\left(R_{n} \rightarrow R_{n+1}\right)}{N\left(R_{n}\right)}
$$

The markov model can be further enhanced by exploiting context information about user's origin $(\mathrm{O})$ and his destination (D). The respective probabilities are given as,

$$
\begin{gathered}
P\left(R_{n} \rightarrow R_{n+1} / O\right)=\frac{N\left(R_{n} \rightarrow R_{n+1} / O\right)}{N\left(R_{n} / O\right)} \\
P\left(R_{n} \rightarrow R_{n+1} / O \& D\right)=\frac{N\left(R_{n} \rightarrow R_{n+1} / O \& D\right)}{N\left(R_{n} / O \& D\right)}
\end{gathered}
$$

In eq.5, user statistics only when he originated from a particular origin are considered, where as, in eq.6 user statistics when originated from a specific origin and traveling to a specific destination are considered for predicting next road (route) of user.

A car traveling at $30 \mathrm{~km} / \mathrm{h}$ is present and it traverses a set of 10 trajectories, each of which includes the 7 landmarks. The car is assumed to follow realistic diurnal mobility [11], where it traverses along a set of fixed trajectories on daily basis instead of following random way point mobility. The base stations are enabled with LTE technology and simulation parameters (e.g., antenna parameters, pathloss models etc.) follow ITU-R, METIS guidelines [12]. The bandwidth is 10 $\mathrm{MHz}$ (50 PRBs) and carrier frequency is $2 \mathrm{GHz}$. A 100 simulation runs are executed to obtain user mobility statistics. Then, aforementioned mobility prediction schemes are carried out for another 100 simulation runs and average accuracy of prediction is calculated. The comparison among different schemes are depicted in figure 3 . It could be observed that prediction accuracy increases when additional context information about user origin and destination are used. The next cell prediction accuracy is enhanced to around $85 \%$ and route prediction accuracy is improved to around $90 \%$, indicating that extracting and exploiting information about user origin and destination from vehicular infrastructure is valuable for mobility prediction.

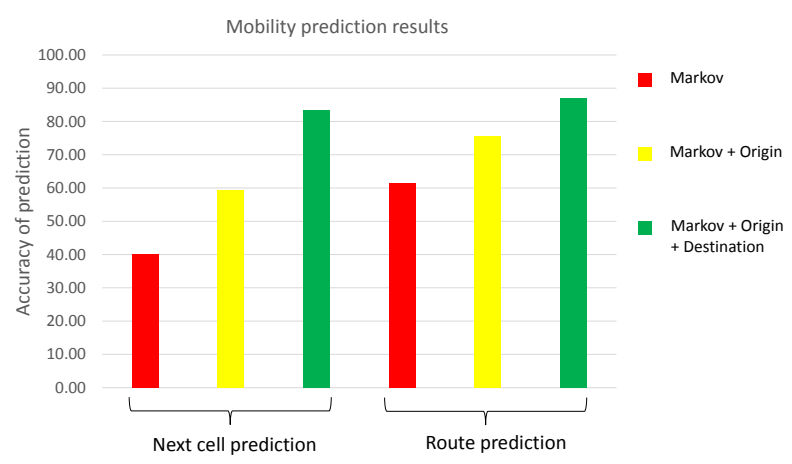

Fig. 3. Mobility Prediction

The mobility prediction is then used in tandem with resource allocation to the user. There are 6 coverage holes (e.g., tunnels) placed in different roads in the scenario and context aware resource allocation algorithm is designed. The user is assumed to be availing buffer-able streaming media service. The idea of the scheme is demonstrated in figure 4 and figure 5 and the algorithm is given below:

1) Check if the user has reached prediction trigger point (predefined point before crossroad).

2) If Yes: Predict the next path, go to step 3.

If No: Go to step 1.

3) Check if there is a coverage hole in predicted path.

4) If Yes: Allocate additional resources to the user and buffer data.

If No: Go to step 1.

5) Check if user actually took the path with coverage hole.

6) If Yes: Continue buffering till user is in coverage hole. If No: Stop buffering, go to step 1.

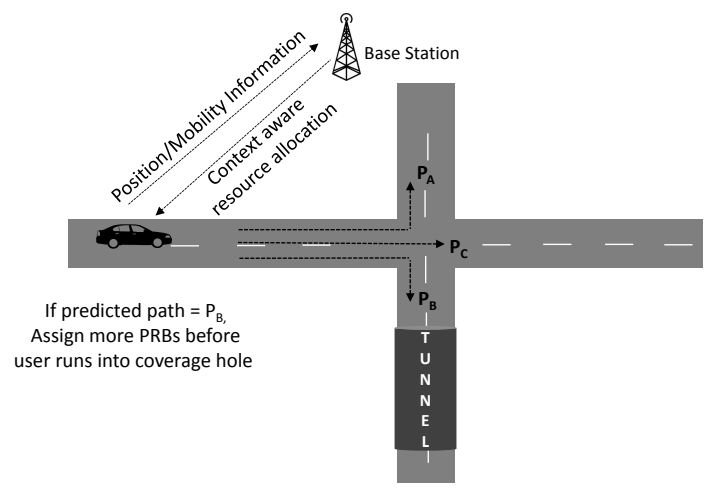

Fig. 4. Context Aware Resource Allocation

A 100 simulation runs are executed to evaluate the average improvement in user throughput when encountered by coverage holes. The results are depicted in figure 6. Improvements 


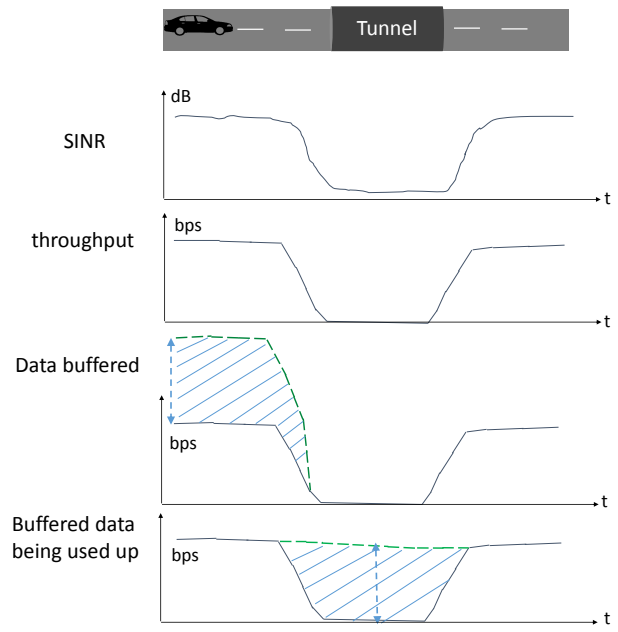

Fig. 5. Using buffered data in coverage holes

of around 35\% and 65\% are observed with allocation of number of physical resource blocks (PRB) of 2 and 3 respectively, triggered by context aware algorithm. The throughput improvements when perfect route knowledge is available are also outlined in figure 6.

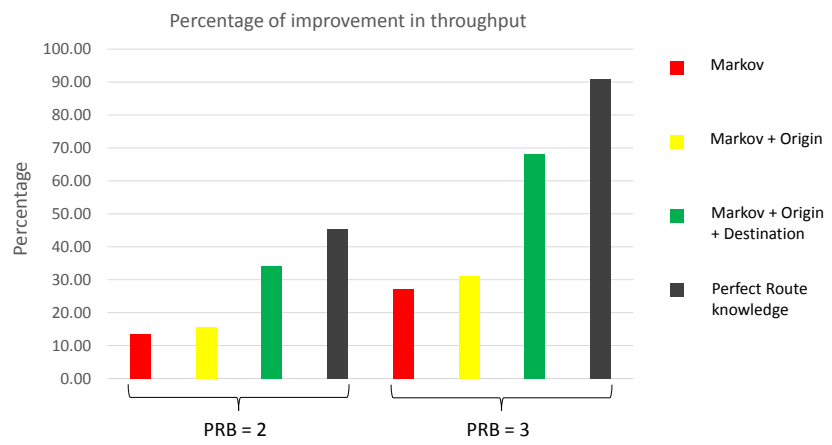

Fig. 6. User throughput improvement in coverage hole

\section{Interfaces to Support Mobility Context Awareness}

Mobility context aware procedures can be functionally decomposed into three blocks as in figure 7 namely:

1) Context extraction: The functionality of obtaining user positions, signal strength/geometry measurements, obtaining information about origin and destination from vehicular infrastructure etc., are inclusive to this block. Velocity estimate is required additionally to set the sampling rate of aforementioned information.

2) Communication: This block deals with signaling various information between user and base stations. Two major parts are: a) Signaling user positions, geometry etc., to the base station. b) Signaling context message (e.g., trigger for load balancing/resource management, cell activation/deactivation message etc.) from serving base station to target (predicted) base station.
3) Prediction/Decision: The prediction of next cell, route and other event predictions making use of extracted context information, take place in this block. The resource management, cell activation/deactivation decisions etc., are also made here.

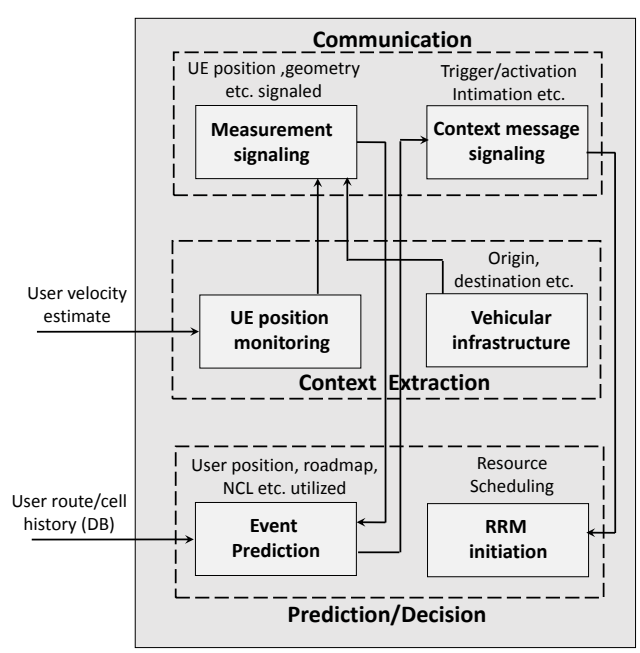

Fig. 7. Functional Decomposition

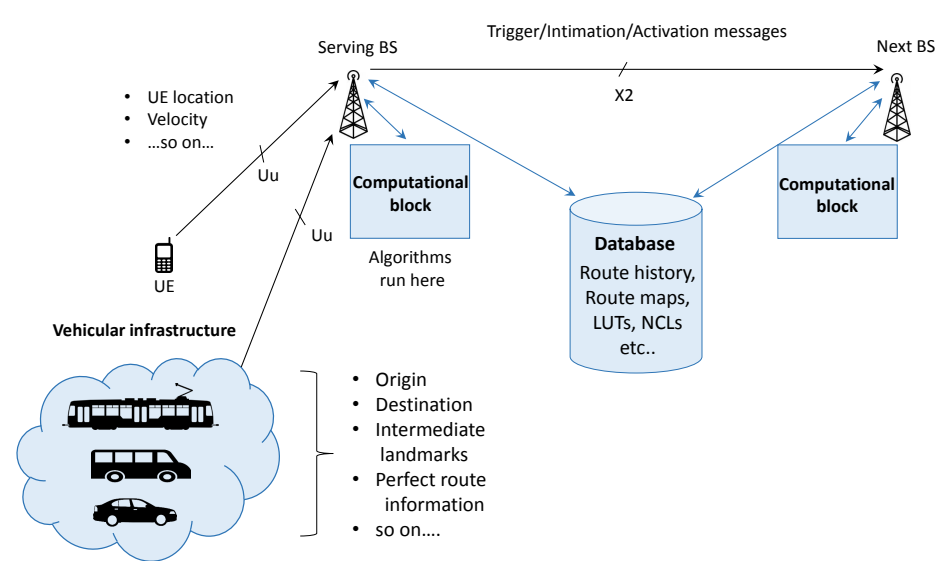

Fig. 8. Overall Architecture

Based on the functional decomposition analysis, the overall architecture to support mobility context awareness is shown in figure 8 . The information about user positions, geometry(dB), velocity etc., need to be signaled to serving base station via Uu interface [13]. Further, information from vehicular infrastructure such as origin, destination, intermediate landmarks, perfect route information (which can become feasible in near future due to advancements in autonomous driving cars) are required to be signaled to serving base station via Uu interface. A database is required to store history of the user mobility, neighboring cell list, route maps of respective regions, related look up tables (LUT) and so on. A proper interface is required to pass information of user mobility to 
database. In this work, mobility statistics of a user are stored in to database via serving base station. The computational blocks at serving base station are responsible for prediction (e.g. next cell) by acquiring information from user and interfacing with database. The subsequent context message (e.g., triggering LB, cell activation) needs to be conveyed to the target cell via X2 interface [13].

From section III-B, it is evident that context information about user origin and destination are valuable for enhancing mobility context awareness. In order to efficiently extract and exploit such information from vehicular infrastructure, new interfaces are required. Figure 9 describes the new interface envisioned between navigation system of the vehicle and the user equipment, to acquire information about origin, destination, route information etc and signal it further to the serving base station via $\mathrm{Uu}$ interface.

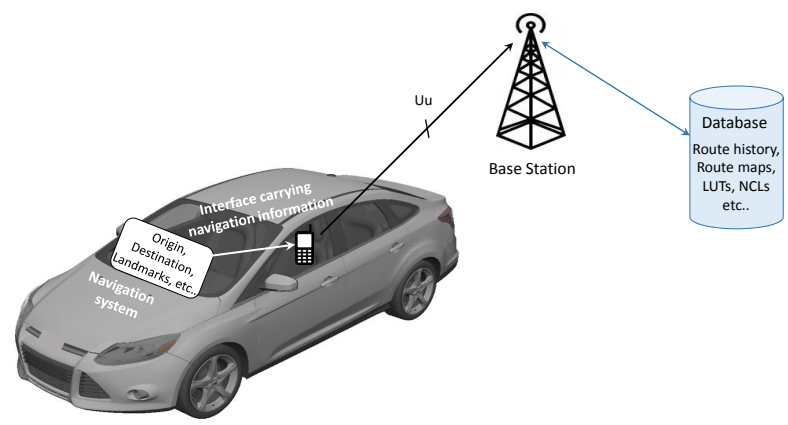

Fig. 9. Interface in Vehicular Infrastructure

In case of several users commuting together (e.g. public transport like bus, train etc.), the number of interfaces and signaling can be minimized by having an anchor node control the users in vehicle, forming a moving network [11]. In such case a new interface is required between anchor node and navigation system as described in figure 10 .

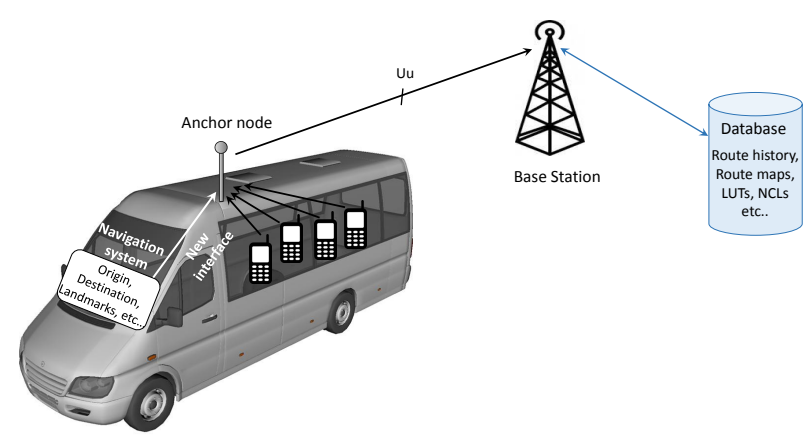

Fig. 10. Interface in Moving Networks

With new interfaces, signaling and functional blocks proposed in figures 8,9 and 10 , it is possible to provide maximum support for mobility context awareness in cellular network.

\section{CONCLUSION AND FUTURE WORK}

Mobile communication has evolved through various generations of technology and is currently in verge of its fifth generation $(5 \mathrm{G})$. The drastic growth of mobile users over last few years has led to a key challenge of atleast 1000 times higher traffic volume by year 2020 than at present. Context awareness is one of the major pillars in $5 \mathrm{G}$ to enable uniform service quality and better QoE, irrespective of high mobility and traffic volume. This paper investigated commonalities among various context awareness schemes with user mobility as key focus, outlined common inputs/information, signaling and interfaces required to support mobility context awareness. Further, context information from vehicular infrastructure were used to enhance mobility context awareness and simulation results demonstrated substantial improvement in user throughput. The new interfaces required in vehicular infrastructure were also discussed and overall architecture to provide support for mobility context awareness was presented. Potential future work is to extend the presented framework to include context awareness schemes focusing device types, applications, service/traffic types etc., along with mobility.

\section{ACKNOWLEDGMENT}

Part of this work has been performed in the framework of FP7 project ICT-317669 METIS II, which is partly funded by the European Union. The authors alone are responsible for the content of the paper.

\section{REFERENCES}

[1] Ericsson, More than 50 billion connected devices, white paper, Feb., 2011.

[2] Nokia Siemens Networks, 2020: Beyond 4G Radio Evolution for the Gigabit Experience, white paper, Aug., 2011.

[3] METIS, D1.1 - Future radio access scenarios, requirements and KPIs, May, 2013, available online: https://www.metis2020.com.

[4] METIS, D4.1 - Summary on preliminary trade-off investigations and first set of potential network-level solutions, Sept., 2013, available online: https://www.metis2020.com.

[5] S. Bellahsene and L. Kloul A New Markov-Based Mobility Prediction Algorithm for Mobile Networks, Conference on Computer Performance Engineering, Bertinoro, Italy, 2010.

[6] A. Hadachi, O. Batrashev et al., Cell Phone Subscribers Mobility Prediction Using Enhanced Markov Chain Algorithm, Intelligent Vehicles Symposium, Michigan, USA, 2014

[7] S.Michaelis, N. Piatkowski and K. Morik Predicting next network cell IDs for moving users with Discriminative and Generative Models, Mobile Data Challenge Workshop in conjunction with International Conference on Pervasive Computing, Newcastle, UK, 2012

[8] K. Laasonen, Clustering and Prediction of Mobile User Routes from Cellular Data, 9th European Conference on Principles and Practice of Knowledge Discovery in Databases (PKDD),Porto, Portugal, 2005

[9] Z. Becvar, P. Mach and B. Simak, Improvement of handover prediction in mobile WiMAX by using two thresholds, The International Journal of Computer and Telecommunications Networking, 2011.

[10] N.P. Kuruvatti; A. Klein; H.D. Schotten, Prediction of Dynamic Crowd Formation in Cellular Networks for Activating Small Cells, VTC-Spring, Glasgow,UK,2015

[11] N.P. Kuruvatti, A. Klein, J. Schneider and H.D. Schotten, Exploiting Diurnal User Mobility for Predicting Cell Transitions, IEEE Globecom workshops, Atlanta, USA, 2013.

[12] METIS, D6.1-Simulation Guidelines, May, 2013, available online: https: //www.metis2020.com.

[13] Alcatel-Lucent, The LTE Network Architecture-A comprehensive tutorial, white paper, Aug., 2009. 